\title{
An Asymmetric Noncommutative Torus
}

\author{
Ludwik DAsBROWSKI ${ }^{\dagger}$ and Andrzej SITARZ $\ddagger$ \\ $\dagger$ SISSA (Scuola Internazionale Superiore di Studi Avanzati), \\ via Bonomea 265, 34136 Trieste, Italy \\ E-mail: dabrow@sissa.it \\ $\ddagger$ Institute of Physics, Jagiellonian University, \\ Stanistawa Eojasiewicza 11, 30-348 Kraków, Poland \\ E-mail: andrzej.sitarz@uj.edu.pl \\ $\S$ Institute of Mathematics of the Polish Academy of Sciences, \\ Śniadeckich 8, 00-656 Warszawa, Poland
}

Received December 09, 2014, in final form September 17, 2015; Published online September 26, 2015 http://dx.doi.org/10.3842/SIGMA.2015.075

\begin{abstract}
We introduce a family of spectral triples that describe the curved noncommutative two-torus. The relevant family of new Dirac operators is given by rescaling one of two terms in the flat Dirac operator. We compute the dressed scalar curvature and show that the Gauss-Bonnet theorem holds (which is not covered by the general result of Connes and Moscovici).
\end{abstract}

Key words: noncommutative geometry; Gauss-Bonnet; spectral triple

2010 Mathematics Subject Classification: 58B34; 46L87

\section{Introduction}

In the seminal works [2, 7] Connes and Tretkoff initiated the investigation of curvature aspects on the noncommutative two torus $\mathbb{T}_{\theta}^{2}$ and have shown the analogue of the Gauss-Bonnet theorem for the conformally rescaled Dirac operator $D$ and the related spin Laplacian corresponding to the standard conformal structure. In [10] these studies were extended to arbitrary conformal structure. The scalar curvature itself was defined and computed in [6] and independently in [11].

The methods used in these papers build on Connes' pseudodifferential calculus [7] and heat kernel short-time asymptotic expansion. The novelty therein is the employment of twisted (or non-unimodular) spectral triples [5], and as a consequence non-tracial weights and the modular operator. For some related papers see $[1,12,13]$.

In the present paper we introduce a family of usual spectral triples in the sense of [4], known also as $K$-cycles [3, Definition IV.2.11], that describe a curved $\mathbb{T}_{\theta}^{2}$. The relevant new Dirac operators correspond to the rescaling one of two terms in the flat Dirac operator by a positive element $k$ from the opposite coordinate algebra in the commutant. This assures that the commutators of $D$ with the elements of the algebra are bounded. Thus, to the best of our knowledge, this provides the first instance of usual spectral triples which describe non flat (curved) noncommutative geometry. It should also be stressed that partial rescalings by $k$ from the algebra itself go outside the realm of spectral triples (even twisted), with geometric meaning (to the best of our knowledge) not yet fully clarified (in this respect see however, e.g., [15]).

Unlike the classical case the new family of Dirac operators is not conformally related to the standard family of "flat" (equivariant) Dirac operators. Since they are not an overall rescaling of any of the so far known Dirac operators one cannot apply the general argument used by Connes 
and Moscovici [6, Theorem 2.1] to establish the Gauss-Bonnet theorem. Thus it is the first instance when one needs to effectively compute it, without certainty that it will be satisfied.

This has been shown perturbatively up to second order in [8] for small modifications of the flat Dirac operator depending on four elements in the opposite algebra. In the present paper we perform the exact computation, which shows that the Gauss-Bonnet theorem indeed extends to a much larger class of spectral triples.

Moreover, we compute the expression for the dressed scalar curvature as an element of the opposite algebra of $\mathbb{T}_{\theta}^{2}$. In the classical case it corresponds to the product of the scalar curvature by the coefficient of the metric volume form with respect to the Lebesgue volume. It should be mentioned that such a dressing is actually tacitly present in all the previous computations.

The results obtained here are possible due to a recent neat generalisation by Lesch [14] of the "rearrangement lemma", which is an important technical tool in [7].

The paper is organised as follows: in Section 2 we briefly present the asymmetric rescaling of the classical flat 2-torus and its curvature. Next we introduce a quantum (noncommutative) analogue of the associated Dirac operator, and the corresponding new family of spectral triples on the noncommutative torus. In Section 3, which is the main part of the paper, we compute the value of the respective zeta function of the new Dirac operators at $z=0$ following the heat trace techniques and pseudodifferential calculus elaborated in [6, 7], and an adaptation of the Lesch rearrangement lemma [14]. In Section 4 we comment on some algebra computations of the (undressed) curvature.

\section{Dirac operator}

Let $\mathbb{T}^{2}$ be the classical torus with coordinates $(x, y) \in[0,2 \pi]^{2}$, equipped with the metric

$$
d x^{2}+k^{-2}(x, y) d y^{2}
$$

where $k$ is a positive function. For example such an "asymmetric torus" metric with

$$
k^{-1}=c+\cos y
$$

comes as the induced metric on the usual realisation of $\mathbb{T}^{2}$ as an embedded surface in $\mathbb{R}^{3}$ with the following parametrisation

$$
X=(c+\cos y) \cos x, \quad Y=(c+\cos y) \sin x, \quad Z=\sin y .
$$

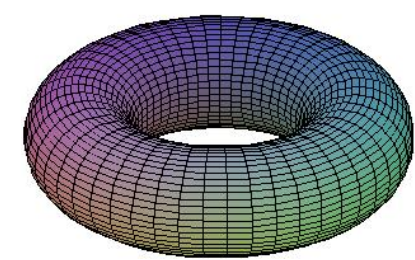

Torus embedded in $\mathbb{R}^{3}$.

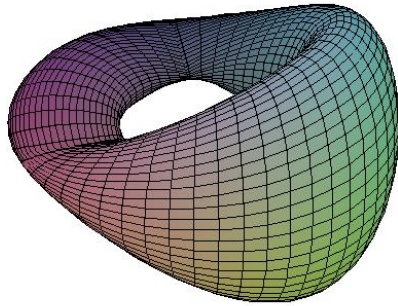

Asymmetric torus in $\mathbb{R}^{3}$.

The scalar curvature of the torus with the metric (2.1) reads

$$
R=2 k^{-1} \partial_{x}^{2}(k)-4 k^{-2}\left(\partial_{x}(k)\right)^{2} .
$$

In the commutative case such metric is, of course, conformally equivalent to some flat metric on the torus even though the explicit formula for the curvature depends on the chosen coordinate 
system. However, when passing to the noncommutative torus we are entering a new unexplored land, where one does not know what is a metric and what is the exact meaning of conformally equivalent. As in the approach of Connes the natural object is the Dirac operator rather than the metric itself. For this reason we propose a new Dirac operator, which generalises to the noncommutative situation the classical case of asymmetric torus.

We start with the commutative case of Dirac operator on $L^{2}\left(\mathbb{T}^{2}, k^{-1} d x d y\right) \otimes \mathbb{C}^{2}$ for the metric (2.1)

$$
\tilde{D}=-i \sigma^{1}\left(\partial_{x}-\frac{1}{2} k^{-1} \partial_{x}(k)\right)-i \sigma^{2} k \partial_{y},
$$

where

$$
\sigma^{1}=\left(\begin{array}{cc}
0 & 1 \\
1 & 0
\end{array}\right), \quad \sigma^{2}=\left(\begin{array}{cc}
0 & -i \\
i & 0
\end{array}\right)
$$

Using the multiplication by $\sqrt{k}$ we obtain a unitarily equivalent self-adjoint Dirac operator

$$
\tilde{D}=-i \sigma^{1} \partial_{x}-i \sigma^{2}\left(k \partial_{y}+\frac{1}{2} \partial_{y}(k)\right)
$$

on the dense domain $H^{1,2}(T)$ in the Hilbert space $L^{2}(T, d x d y) \otimes \mathbb{C}^{2}$.

Next we pass to the noncommutative torus $\mathbb{T}_{\theta}^{2}$ [3], about which we collected the needed information in Appendix A. In particular we shall use therein the usual trace $\mathfrak{t}$, the usual selfadjoint derivations $\delta_{1}, \delta_{2}$, and the standard real structure (Tomita-Takesaki conjugation) $J$ on the coordinate algebra $A\left(\mathbb{T}_{\theta}^{2}\right)$. Then we introduce the following new family of spectral triples, which is an immediate noncommutative analogue of (2.3).

Definition 2.1. For $k \in J A\left(\mathbb{T}_{\theta}^{2}\right) J^{-1}$ we set

$$
D_{k}=\sigma^{1} \delta_{1}+\sigma^{2}\left(k \delta_{2}+\frac{1}{2} \delta_{2}(k)\right)
$$

acting on $\mathcal{H}=L^{2}\left(\mathbb{T}_{\theta}^{2}, \mathfrak{t}\right) \otimes \mathbb{C}^{2}$, obtaining a family of spectral triples $\left(\mathbb{T}_{\theta}^{2}, \mathcal{H}, D_{k}\right)$.

The form of the operator $D_{k}$ resembles the classical Dirac operator $D(2.3)$, however with the partial derivatives replaced by $i \delta_{1}, i \delta_{2}$. Since we take $k$ in the algebra $J A\left(\mathbb{T}_{\theta}^{2}\right) J^{-1}$ commuting with $A\left(\mathbb{T}_{\theta}^{2}\right)$ due to the property (A.1), $D_{k}$ has bounded commutators with $a \in A\left(\mathbb{T}_{\theta}^{2}\right)$. Next the self-adjointness and the compact resolvent property follow from the fact that $D_{k}$ is a perturbation of the flat Dirac operator $D$ (corresponding to $k=1$ ) by a (self-adjoint) bounded operator. Thus it defines a spectral triple in the usual sense.

Furthermore, $D_{k}$ is a differential operator in the sense of [7] and we can extract the associated (dressed) scalar curvature following the methods introduced and applied to the global conformal rescaling of the noncommutative $[6,7]$. The idea is to look for the functional:

$$
f \mapsto a_{2}\left(D_{k} ; f\right)
$$

where $f$ is an element of $J A\left(\mathbb{T}_{\theta}^{2}\right) J^{-1}$ (or $A\left(\mathbb{T}_{\theta}^{2}\right)$ ) and $a_{2}\left(D_{k} ; f\right)$ is the second heat trace coefficient of $\operatorname{Tr}\left(f e^{-t D_{k}^{2}}\right)$. Since the computations which use the pseudodifferential operators allow us to express the results in terms of $\tau(f \widetilde{R})$ for an element $\widetilde{R}$ of the algebra $J A\left(\mathbb{T}_{\theta}^{2}\right) J^{-1}$, one can interpret it as the dressed scalar curvature, which in the classical case equals $\widetilde{R}=\sqrt{g} R$.

The noncommutative case is, in fact, much richer, since the chiral heat trace functional $\operatorname{Tr}\left(f \gamma e^{-t D_{k}^{2}}\right)$ does not vanish identically. Using similar arguments as above we can introduce the dressed chiral scalar curvature, $\widetilde{R_{\gamma}}$ as an element of $J A\left(\mathbb{T}_{\theta}^{2}\right) J^{-1}$ such that the heat trace coefficient $a_{2}\left(D_{k}, f \gamma\right)$ is expressed as $\tau\left(f \widetilde{R}_{\gamma}\right)$. 


\section{The curvature}

The square of $D$ reads

$$
\begin{aligned}
\left(D_{k}\right)^{2}= & \left(\delta_{1}\right)^{2}+k^{2}\left(\delta_{2}\right)^{2}+\left(\frac{3}{2} k \delta_{2}(k)+\frac{1}{2} \delta_{2}(k) k+\sigma^{1} \sigma^{2} \delta_{1}(k)\right) \delta_{2} \\
& +\frac{1}{4}\left(\delta_{2}(k)\right)^{2}+\frac{1}{2} \sigma^{1} \sigma^{2} \delta_{12}(k)+\frac{1}{2} k \delta_{22}(k),
\end{aligned}
$$

and its symbol is

$$
\sigma\left(\left(D_{k}\right)^{2}\right)=a_{0}+a_{1}+a_{2},
$$

where

$$
\begin{aligned}
& a_{0}=\xi_{1}^{2}+k^{2} \xi_{2}^{2}, \quad a_{1}=\left(\frac{3}{2} k \delta_{2}(k)+\frac{1}{2} \delta_{2}(k) k+\sigma^{1} \sigma^{2} \delta_{1}(k)\right) \xi_{2}, \\
& a_{2}=\frac{1}{4}\left(\delta_{2}(k)\right)^{2}+\frac{1}{2} \sigma^{1} \sigma^{2} \delta_{12}(k)+\frac{1}{2} k \delta_{22}(k) .
\end{aligned}
$$

As was demonstrated first in [7] the value $\zeta(0)$ at the origin of the zeta function of the operator $\left(D_{k}\right)^{2}$ is given by

$$
\zeta(0)=-\int \mathfrak{t}\left(b_{2}(\xi)\right) d \xi
$$

where $b_{2}(\xi)$ is a symbol of order -4 of the pseudodifferential operator $\left(\left(D_{k}\right)^{2}+1\right)^{-1}$. Here we assume that $\left(D_{k}\right)$ has no zero eigenvalues, which is satisfied for generic $k$. Otherwise, in the case that the Dirac operator has 0 as an eigenvalue, a constant term equal to $\operatorname{dim}\left(\operatorname{ker}\left(D_{k}\right)\right)$ is present. The term on the right hand side is interpreted as the quantum analogue of the integrated scalar curvature dressed by the square root of the determinant of the metric tensor.

The symbol $b_{2}(\xi)$ can be computed by pseudodifferential calculus of symbols from the symbol $a_{2}(\xi)+a_{1}(\xi)+a_{0}(\xi)$ of $\left(D_{k}\right)^{2}$ as follows

$$
\begin{aligned}
b_{0}= & \left(a_{2}+1\right)^{-1}, \quad b_{1}=-\left(b_{0} a_{1} b_{0}+\partial_{1}\left(b_{0}\right) \delta_{1}\left(a_{2}\right) b_{0}+\partial_{2}\left(b_{0}\right) \delta_{2}\left(a_{2}\right) b_{0}\right), \\
b_{2}= & -\left(b_{0} a_{0} b_{0}+b_{1} a_{1} b_{0}+\partial_{1}\left(b_{0}\right) \delta_{1}\left(a_{1}\right) b_{0}+\partial_{2}\left(b_{0}\right) \delta_{2}\left(a_{1}\right) b_{0}+\partial_{1}\left(b_{1}\right) \delta_{1}\left(a_{2}\right) b_{0}\right. \\
& \left.+\partial_{2}\left(b_{1}\right) \delta_{2}\left(a_{2}\right) b_{0}+\frac{1}{2} \partial_{11}\left(b_{0}\right) \delta_{1}^{2}\left(a_{2}\right) b_{0}+\frac{1}{2} \partial_{22}\left(b_{0}\right) \delta_{2}^{2}\left(a_{2}\right) b_{0}+\partial_{12}\left(b_{0}\right) \delta_{12}\left(a_{2}\right) b_{0}\right) .
\end{aligned}
$$

Since to obtain the curvature (or $\zeta(0)$ ) we need to integrate with respect to $\xi_{1}, \xi_{2}$, we notice that all the terms which contain odd powers of these variables shall vanish. Therefore, we can neglect them and keep for computations only the relevant parts with even powers. They read then as follows

$$
b_{2}^{\text {even }}=A+B+C,
$$

where

$$
\begin{aligned}
A= & -2 k b_{0}^{2} \delta_{1}(k) k b_{0} \delta_{1}(k) b_{0} \xi_{2}^{4}+4 k b_{0}^{2} \delta_{1}(k) k b_{0}^{2} \delta_{1}(k) b_{0} \xi_{1}^{2} \xi_{2}^{4}-2 k b_{0}^{2} \delta_{1}(k) b_{0} \delta_{1}(k) k b_{0} \xi_{2}^{4} \\
& +4 k b_{0}^{2} \delta_{1}(k) b_{0}^{2} \delta_{1}(k) k b_{0} \xi_{1}^{2} \xi_{2}^{4}+8 k b_{0}^{3} \delta_{1}(k) k b_{0} \delta_{1}(k) b_{0} \xi_{1}^{2} \xi_{2}^{4}+8 k b_{0}^{3} \delta_{1}(k) b_{0} \delta_{1}(k) k b_{0} \xi_{1}^{2} \xi_{2}^{4} \\
& -b_{0} \delta_{1}(k) b_{0} \delta_{1}(k) b_{0} \xi_{2}^{2}+2 b_{0}^{2} \delta_{1}(k) \delta_{1}(k) b_{0} \xi_{2}^{2}-2 b_{0}^{2} \delta_{1}(k) k b_{0} \delta_{1}(k) k b_{0} \xi_{2}^{4} \\
& +4 b_{0}^{2} \delta_{1}(k) k b_{0}^{2} \delta_{1}(k) k b_{0} \xi_{1}^{2} \xi_{2}^{4}-2 b_{0}^{2} \delta_{1}(k) k^{2} b_{0} \delta_{1}(k) b_{0} \xi_{2}^{4}+4 b_{0}^{2} \delta_{1}(k) k^{2} b_{0}^{2} \delta_{1}(k) b_{0} \xi_{1}^{2} \xi_{2}^{4} \\
& -8 b_{0}^{3} \delta_{1}(k) \delta_{1}(k) b_{0} \xi_{1}^{2} \xi_{2}^{2}+8 b_{0}^{3} \delta_{1}(k) k b_{0} \delta_{1}(k) k b_{0} \xi_{1}^{2} \xi_{2}^{4}+8 b_{0}^{3} \delta_{1}(k) k^{2} b_{0} \delta_{1}(k) b_{0} \xi_{1}^{2} \xi_{2}^{4}, \\
B= & \frac{15}{4} k b_{0} \delta_{2}(k) k b_{0} \delta_{2}(k) b_{0} \xi_{2}^{2}-3 k b_{0} \delta_{2}(k) k^{2} b_{0}^{2} \delta_{2}(k) k b_{0} \xi_{2}^{4}-3 k b_{0} \delta_{2}(k) k^{3} b_{0}^{2} \delta_{2}(k) b_{0} \xi_{2}^{4} \\
& +\frac{9}{4} k b_{0} \delta_{2}(k) b_{0} \delta_{2}(k) k b_{0} \xi_{2}^{2}+6 k^{2} b_{0}^{2} \delta_{2}(k) \delta_{2}(k) b_{0} \xi_{2}^{2}-8 k^{2} b_{0}^{2} \delta_{2}(k) k b_{0} \delta_{2}(k) k b_{0} \xi_{2}^{4}
\end{aligned}
$$




$$
\begin{aligned}
& -10 k^{2} b_{0}^{2} \delta_{2}(k) k^{2} b_{0} \delta_{2}(k) b_{0} \xi_{2}^{4}+4 k^{2} b_{0}^{2} \delta_{2}(k) k^{3} b_{0}^{2} \delta_{2}(k) k b_{0} \xi_{2}^{6}+4 k^{2} b_{0}^{2} \delta_{2}(k) k^{4} b_{0}^{2} \delta_{2}(k) b_{0} \xi_{2}^{6} \\
& -12 k^{3} b_{0}^{2} \delta_{2}(k) k b_{0} \delta_{2}(k) b_{0} \xi_{2}^{4}+4 k^{3} b_{0}^{2} \delta_{2}(k) k^{2} b_{0}^{2} \delta_{2}(k) k b_{0} \xi_{2}^{6}+4 k^{3} b_{0}^{2} \delta_{2}(k) k^{3} b_{0}^{2} \delta_{2}(k) b_{0} \xi_{2}^{6} \\
& -10 k^{3} b_{0}^{2} \delta_{2}(k) b_{0} \delta_{2}(k) k b_{0} \xi_{2}^{4}-8 k^{4} b_{0}^{3} \delta_{2}(k) \delta_{2}(k) b_{0} \xi_{2}^{4}+8 k^{4} b_{0}^{3} \delta_{2}(k) k b_{0} \delta_{2}(k) k b_{0} \xi_{2}^{6} \\
& +8 k^{4} b_{0}^{3} \delta_{2}(k) k^{2} b_{0} \delta_{2}(k) b_{0} \xi_{2}^{6}+8 k^{5} b_{0}^{3} \delta_{2}(k) k b_{0} \delta_{2}(k) b_{0} \xi_{2}^{6}+8 k^{5} b_{0}^{3} \delta_{2}(k) b_{0} \delta_{2}(k) k b_{0} \xi_{2}^{6} \\
& -\frac{1}{4} b_{0} \delta_{2}(k) \delta_{2}(k) b_{0}+\frac{3}{4} b_{0} \delta_{2}(k) k b_{0} \delta_{2}(k) k b_{0} \xi_{2}^{2}+\frac{5}{4} b_{0} \delta_{2}(k) k^{2} b_{0} \delta_{2}(k) b_{0} \xi_{2}^{2} \\
& -b_{0} \delta_{2}(k) k^{3} b_{0}^{2} \delta_{2}(k) k b_{0} \xi_{2}^{4}-b_{0} \delta_{2}(k) k^{4} b_{0}^{2} \delta_{2}(k) b_{0} \xi_{2}^{4},
\end{aligned}
$$

and

$$
\begin{aligned}
C= & k b_{0}^{2} \delta_{11}(k) b_{0} \xi_{2}^{2}-4 k b_{0}^{3} \delta_{11}(k) b_{0} \xi_{1}^{2} \xi_{2}^{2}+b_{0}^{2} \delta_{11}(k) k b_{0} \xi_{2}^{2}-4 b_{0}^{3} \delta_{11}(k) k b_{0} \xi_{1}^{2} \xi_{2}^{2}-\frac{1}{2} k b_{0} \delta_{22}(k) b_{0} \\
& +2 k^{2} b_{0}^{2} \delta_{22}(k) k b_{0} \xi_{2}^{2}+4 k^{3} b_{0}^{2} \delta_{22}(k) b_{0} \xi_{2}^{2}-4 k^{4} b_{0}^{3} \delta_{22}(k) k b_{0} \xi_{2}^{4}-4 k^{5} b_{0}^{3} \delta_{22}(k) b_{0} \xi_{2}^{4} .
\end{aligned}
$$

Similarly for the chiral part $\widetilde{R}_{\gamma}$ of the curvature, we have

$$
b_{\gamma 2}^{\text {even }}=A_{\gamma}+B_{\gamma}+C_{\gamma},
$$

where

$$
\begin{aligned}
A_{\gamma}= & -2 k^{2} b_{0}^{2} \delta_{1}(k) k b_{0} \delta_{2}(k) b_{0} i \xi_{2}^{4}-2 k^{2} b_{0}^{2} \delta_{1}(k) b_{0} \delta_{2}(k) k b_{0} i \xi_{2}^{4}+\frac{5}{2} b_{0} \delta_{1}(k) k b_{0} \delta_{2}(k) b_{0} i \xi_{2}^{2} \\
& -2 b_{0} \delta_{1}(k) k^{2} b_{0}^{2} \delta_{2}(k) k b_{0} i \xi_{2}^{4}-2 b_{0} \delta_{1}(k) k^{3} b_{0}^{2} \delta_{2}(k) b_{0} i \xi_{2}^{4}+\frac{3}{2} b_{0} \delta_{1}(k) b_{0} \delta_{2}(k) k b_{0} i \xi_{2}^{2}, \\
B_{\gamma}= & \frac{3}{2} k b_{0} \delta_{2}(k) b_{0} \delta_{1}(k) b_{0} i \xi_{2}^{2}-2 k^{2} b_{0}^{2} \delta_{2}(k) k b_{0} \delta_{1}(k) b_{0} i \xi_{2}^{4} \\
& -2 k^{3} b_{0}^{2} \delta_{2}(k) b_{0} \delta_{1}(k) b_{0} i \xi_{2}^{4}+\frac{1}{2} b_{0} \delta_{2}(k) k b_{0} \delta_{1}(k) b_{0} i \xi_{2}^{2},
\end{aligned}
$$

and

$$
C_{\gamma}=2 k^{2} b_{0}^{2} \delta_{12}(k) b_{0} i \xi_{2}^{2}-\frac{1}{2} b_{0} \delta_{12}(k) b_{0} i
$$

\subsection{The classical limit}

At this point we can check the classical (commutative) value of our expressions for $\theta=0$. The symbol $b_{2}$ becomes

$$
\begin{aligned}
b_{2}(\xi)= & 48 b_{0}^{5} k^{6} \delta_{2}(k)^{2} \xi_{2}^{6}+48 b_{0}^{5} k^{2} \delta_{1}(k)^{2} \xi_{1}^{2} \xi_{2}^{4}-8 b_{0}^{4} k^{5} \delta_{22}(k) \xi_{2}^{4}-56 b_{0}^{4} k^{4} \delta_{2}(k)^{2} \xi_{2}^{4} \\
& -8 b_{0}^{4} k^{2} \delta_{1}(k)^{2} \xi_{2}^{4}-8 b_{0}^{4} k \delta_{11}(k) \xi_{1}^{2} \xi_{2}^{2}-8 b_{0}^{4} \delta_{1}(k)^{2} \xi_{1}^{2} \xi_{2}^{2}+6 b_{0}^{3} k^{3} \delta_{22}(k) \xi_{2}^{2} \\
& +14 b_{0}^{3} k^{2} \delta_{2}(k)^{2} \xi_{2}^{2}+2 b_{0}^{3} k \delta_{11}(k) \xi_{2}^{2}+b_{0}^{3} \delta_{1}(k)^{2} \xi_{2}^{2}-\frac{1}{2} b_{0}^{2} k \delta_{22}(k)-\frac{1}{4} b_{0}^{2} \delta_{2}(k)^{2}
\end{aligned}
$$

which after integration gives

$$
\int b_{2} d \xi_{1} d \xi_{2}=-\frac{\pi}{3} \frac{\left(\delta_{1}(k)\right)^{2}}{k^{3}}+\frac{\pi}{6} \frac{\delta_{11}(k)}{k^{2}} .
$$

Taking into account that we compute the Gilkey-Seeley-deWitt coefficients of the heat kernel asymptotic for the square of the Dirac operator and not the Laplace operator itself, and assuming as above that $D_{k}$ has no zero eigenvalues, we have

$$
\zeta(0)=\frac{1}{48 \pi} \int \sqrt{g} R .
$$

The assumption that 0 is not in the spectrum of $D_{k}$, is satisfied for generic $k$. Otherwise, in the case that $D_{k}$ has 0 as an eigenvalue, there is a constant term contribution equal to $\operatorname{dim}\left(\operatorname{ker} D_{k}\right)$. 
Moreover, since $\mathfrak{t}=\frac{1}{4 \pi^{2}} \int d x d y$ for $\theta=0$, taking into account the appropriate rescaling of the volume form and putting it all together we obtain

$$
\sqrt{g} R=48 \pi \frac{1}{4 \pi^{2}}\left(-\frac{\pi}{3} \frac{\left(\partial_{1}(k)\right)^{2}}{k^{3}}+\frac{\pi}{6} \frac{\partial_{11}(k)}{k^{2}}\right)=\left(2 k^{-2} \partial_{11}(k)-4 k^{-3}\left(\partial_{1}(k)\right)^{2}\right),
$$

which agrees with the classical formula (2.2). Similarly, the symbol $b_{\gamma 2}$ becomes

$$
b_{\gamma 2}(\xi)=-12 b_{0}^{4} k^{3} \delta_{1}(k) \delta_{2}(k) i \xi_{2}^{4}+2 b_{0}^{3} k^{2} \delta_{12}(k) i \xi_{2}^{2}+6 b_{0}^{3} k \delta_{1}(k) \delta_{2}(k) i \xi_{2}^{2}-\frac{1}{2} b_{0}^{2} \delta_{12}(k) i,
$$

which after integration yields

$$
\int d \xi_{1} d \xi_{2} b_{\gamma 2}=0
$$

and thus

$$
\sqrt{g} R_{\gamma}=0
$$

Before we can proceed with the noncommutative computation let us recall the general framework of computations as shown recently by Lesch [14].

\subsection{Rearrangement lemma}

In [14] Lesch proved the following formula (with a slightly modified notation)

$$
\begin{aligned}
\int_{0}^{\infty} & f_{0}\left(u k^{2}\right) a_{1} f_{1}\left(u k^{2}\right) a_{2} \cdots a_{p} f_{p}\left(u k^{2}\right) d u \\
& =k^{-2} m F\left(\Delta_{2}^{(1)}, \Delta_{2}^{(1)} \Delta_{2}^{(2)}, \ldots, \Delta_{2}^{(1)} \cdots \Delta_{2}^{(p)}\right)\left(a_{1} \otimes a_{2} \otimes \cdots \otimes a_{p}\right),
\end{aligned}
$$

where $m$ is the $p$-fold multiplication, the function $F$ of $p$ variables reads

$$
F\left(s_{1}, \ldots, s_{p}\right)=\int_{0}^{\infty} f_{0}(u) f_{1}\left(u s_{1}\right) \cdots f_{p}\left(u s_{p}\right) d u
$$

and $\Delta_{2}^{(j)}$ signifies the square of the modular operator $\Delta, \Delta(a)=k^{-1} a k$, acting on the $j$-th factor $a_{j}$ in the $p$-fold tensor product product $a_{1} \otimes a_{2} \otimes \cdots \otimes a_{p}$.

In our case we need to adapt the formula to a slightly different setting, when we integrate over two variables $\xi_{1}$ and $\xi_{2}$. All the integrals we have are of the form

$$
\mathcal{J}=\int_{-\infty}^{\infty} d \xi_{1} \int_{-\infty}^{\infty} d \xi_{2} k^{n_{1}} b_{0}^{m_{1}}\left(\xi_{1}, \xi_{2}\right) X k^{n_{2}} b_{0}^{m_{2}}\left(\xi_{1}, \xi_{2}\right) Y k^{n_{3}} b_{0}^{m_{3}}\left(\xi_{1}, \xi_{2}\right) \xi_{1}^{2 k_{1}} \xi_{2}^{2 k_{2}},
$$

where $X, Y$ are some derivatives of $k$ and

$$
b_{0}\left(\xi_{1}, \xi_{2}\right)=\frac{1}{1+\xi_{1}^{2}+k^{2} \xi^{2}} .
$$

Extending the result of Lesch we see that

$$
\mathcal{J}=F\left(\Delta^{(1)}, \Delta^{(1)} \Delta^{(2)}\right)(X \cdot Y),
$$

where after change of variables we obtain

$$
F(s, t)=2 \int_{0}^{\infty} d v \int_{0}^{\infty} d u k^{n_{1}+n_{2}+n 3-1-2 k_{2}} \frac{u^{k_{2}-\frac{1}{2}} v^{2 k_{1}}}{\left(1+v^{2}+u\right)^{m_{1}}} \frac{s^{n_{2}}}{\left(1+v^{2}+u s^{2}\right)^{m_{2}}} \frac{t^{n_{3}}}{\left(1+v^{2}+u t^{2}\right)^{m_{3}}} .
$$

In case $Y=1$ the resulting function depends only on $s$. 


\subsection{The curvature and its trace}

In order to compute explicitly the expressions for the curvature we shall use the following lemma.

Lemma 3.1. Under the trace an entire function $F$ of two variables satisfies

$$
\mathfrak{t}\left(F\left(\Delta^{(1)}, \Delta^{(1)} \Delta^{(2)}\right)(X \cdot Y)\right)=\mathfrak{t}\left(F\left(\Delta^{(1)}, \mathrm{id}\right)(X Y)\right)=\mathfrak{t}\left(F\left(\Delta^{(1)}, 1\right)(X) Y\right) .
$$

and in case of one variable

$$
\mathfrak{t}\left(F\left(\Delta^{(1)}\right)(X)\right)=\mathfrak{t}(F(1) X) .
$$

Proof. We have

$$
F(s, t)=\sum_{n, m \geq 0} f_{n m} s^{n} t^{m}
$$

so

$$
\begin{aligned}
\mathfrak{t}(F & \left.\left(\Delta^{(1)}, \Delta^{(1)} \Delta^{(2)}\right)(X \cdot Y)\right)=\sum_{n, m \geq 0} f_{n m} \mathfrak{t}\left(\Delta^{n+m}(X) \Delta^{m}(Y)\right) \\
& =\sum_{n, m \geq 0} f_{n m} \mathfrak{t}\left(\Delta^{m}\left(\Delta^{n}(X) Y\right)\right)=\sum_{n, m \geq 0} f_{n m} \mathfrak{t}\left(\Delta^{n}(X) Y\right)=\mathfrak{t}\left(F\left(\Delta^{(1)}, 1\right)(X \cdot Y)\right) .
\end{aligned}
$$

The other identity is a simple consequence of the above one.

\subsection{Curvature and chiral curvature}

We can present the first main result on the dressed scalar curvature of the asymmetric noncommutative torus:

Proposition 3.2. The dressed scalar curvature for the asymmetric torus is

$$
\begin{aligned}
\widetilde{R}= & F_{11}\left(\Delta^{(1)}, \Delta^{(1)} \Delta^{(2)}\right)\left(\delta_{1}(k) \cdot \delta_{1}(k)\right)+F_{22}\left(\Delta^{(1)}, \Delta^{(1)} \Delta^{(2)}\right)\left(\delta_{2}(k), \delta_{2}(k)\right) \\
& +F_{11}^{\prime}\left(\Delta^{(1)}\right)\left(\delta_{1}(k)^{2}\right)+F_{22}^{\prime}\left(\Delta^{(1)}\right)\left(\delta_{2}(k)^{2}\right)+F_{1}\left(\Delta^{(1)}\right)\left(\delta_{11}(k)\right)+F_{2}\left(\Delta^{(1)}\right)\left(\delta_{22}(k)\right),
\end{aligned}
$$

where

$$
\begin{aligned}
& F_{11}(s, t)=-\frac{2 \pi}{3 k^{3}} \frac{\left(2 s^{2}+4 s t+4 s+3+8 t+3 t^{2}\right)}{(t+1)^{3}(s+1)(s+t)}, \quad F_{22}(s, t)=\frac{\pi}{2 k} \frac{\left(t^{2}-6 t+1\right)}{(t+1)^{3}}, \\
& F_{11}^{\prime}(s)=\frac{4 \pi}{3 k^{3}} \frac{1}{(s+1)^{3}}, \quad F_{22}^{\prime}(s)=-\frac{\pi}{2 k} \frac{\left(s^{2}-6 s+1\right)}{(s+1)^{3}},
\end{aligned}
$$

and

$$
F_{1}(s)=\frac{2 \pi}{3 k^{2}} \frac{1}{(s+1)^{2}}, \quad F_{2}(s)=0 .
$$

The trace of $\widetilde{R}$ vanishes.

Proof. First of all, observe that

$$
F_{22}(s, 1)+F_{22}^{\prime}(1)=0, \quad F_{2}(1)=0,
$$

so all terms containing $\delta_{2}(k)$ and $\delta_{22}(k)$ vanish. 
For the terms containing $\delta_{1}(k)$ we have

$$
F_{11}(s, 1)+F_{11}^{\prime}(1)=-\frac{\pi}{3 k^{3}} \frac{s+3}{(s+1)^{2}} .
$$

Then using the identity

$$
\mathfrak{t}\left(k^{-2} \delta_{11}(k)\right)=2 \mathfrak{t}\left(k^{-2} \delta_{1}(k) k^{-1} \delta_{1}(k)\right)=2 \mathfrak{t}\left(k^{-3} \Delta^{-1}\left(\delta_{1}(k)\right) \delta_{1}(k)\right),
$$

which follows directly from the Leibniz rule and the fact that the trace is closed, we can rewrite the trace of the sum of the remaining terms $F_{11}, F_{11}^{\prime}$ and $F_{1}$ as

$$
\mathfrak{t}\left(k^{-3} H(\Delta)\left(\delta_{1}(k)\right) \delta_{1}(k)\right),
$$

where

$$
H(s)=\frac{\pi}{3 k^{3}} \frac{1-s}{s(s+1)^{2}} .
$$

Next, we observe that for any $A$ and $B$ and an entire function $H$

$$
\mathfrak{t}\left(k^{-3} H(\Delta)(A) B\right)=\mathfrak{t}\left(H(\Delta)\left(\Delta^{3}(A)\right) k^{-3} B\right)=\mathfrak{t}\left(k^{-3} B H(\Delta)\left(\Delta^{3}(A)\right)\right),
$$

and

$$
\mathfrak{t}\left(k^{-3} H(\Delta)(A) B\right)=\mathfrak{t}\left(k^{-3} A H\left(\Delta^{-1}\right)(B)\right) .
$$

Now if $A=B$ then both expressions on the right-hand side are identical. In our case, however

$$
H(s) s^{3}=\frac{\pi}{3 k^{3}} \frac{s^{2}(1-s)}{(s+1)^{2}} \quad \text { and } \quad H\left(s^{-1}\right)=\frac{\pi}{3 k^{3}} \frac{s^{2}(s-1)}{(s+1)^{2}},
$$

and therefore since

$$
H(s) s^{3}=-H\left(s^{-1}\right)
$$

the trace of the above expression must vanish, hence, the Gauss-Bonnet theorem holds.

Next we give the second main result on the dressed chiral curvature of the asymmetric noncommutative torus:

Proposition 3.3. The dressed chiral curvature for the asymmetric torus is

$$
\begin{aligned}
\widetilde{R}_{\gamma}= & G_{12}\left(\Delta^{(1)}, \Delta^{(1)} \Delta^{(2)}\right)\left(\delta_{1}(k), \delta_{2}(k)\right) \\
& +G_{21}\left(\Delta^{(1)}, \Delta^{(1)} \Delta^{(2)}\right)\left(\delta_{2}(k), \delta_{1}(k)\right)+G\left(\Delta^{(1)}\right)\left(\delta_{12}(k)\right),
\end{aligned}
$$

where

$$
G_{12}(s, t)=\frac{\pi}{k^{2}} \frac{(t-1)}{(t+1)^{2}(s+1)}, \quad G_{21}(s, t)=\frac{\pi}{k^{2}} \frac{(t-1)}{(t+1)^{2}(s+t)}, \quad G(s)=-\frac{\pi}{k} \frac{(s-1)}{(s+1)^{2}} .
$$

The trace of $R_{\gamma}$ vanishes.

Proof. By computation. Then the last statement follows from

$$
G_{12}(s, 1)=G_{21}(s, 1)=G(1)=0 .
$$




\section{Final comments}

We have introduced a new class of spectral triples that describe a curved geometry of the noncommutative torus. The associated Dirac operators are obtained through a partial rescaling of the flat Dirac operator by elements from the opposite coordinate algebra. It turns out that the dressed scalar curvature does not vanish, but the Gauss-Bonnet theorem holds for that family.

Let us stress that even though in the classical limit they arise from the metric, which is conformally equivalent to the flat one, this is an open problem in the noncommutative situation. An interesting question is if and in which sense this can be established also in the noncommutative case.

It becomes now more evident that the class of admissible Dirac operators on the noncommutative torus is certainly bigger than the one-parameter family of "flat metric" (equivariant) Dirac operators. It is therefore necessary to study the conditions and the general setup of such construction, and we conjecture that the class should contain all operators proposed in [8] and their fluctuations.

Although in this paper we have concentrated on the 2-dimensional case, it is natural to consider generalisations of the result to 3 and more dimensions. In particular it is interesting to study the curvature and minimality of yet another class Dirac operators introduced in [9], which are similar to the ones encountered here.

Finally, we can comment on the purely algebraic computation by Rosenberg [16] of the curvature and Gauss-Bonnet term for the conformally rescaled metric on the noncommutative torus using the noncommutative notion of Levi-Civita connection. His method can be extended to the case we consider and a direct computation yields the Gauss-Bonnet functional

$$
2 \mathfrak{t}\left(-\frac{9}{4} k^{-2} \delta_{1}(k) k^{-1} \delta_{1}(k)+\frac{1}{4} k^{-3} \delta_{1}(k) \delta_{1}(k)+k^{-2} \delta_{11}(k)\right),
$$

which however does not vanish, and the Gauss-Bonnet theorem does not hold.

Instead there is an even simpler possibility that yields an algebraic expression for a candidate for the scalar curvature. Namely, we note that the formula (2.1) in [8] for the classical scalar curvature in terms of the orthonormal basis of vector fields (moving frame or two-bein) for the partially rescaled metric, makes a perfect sense also for the noncommutative torus (there is no ordering ambiguity). Taking the two-bein as $\left(\delta_{1}, k \delta_{2}\right)$ one obtains

$$
\mathfrak{t}\left(-4 k^{-2} \delta_{1}(k) k^{-1} \delta_{1}(k)+2 k^{-2} \delta_{11}(k)\right),
$$

which vanishes due to the properties of the trace.

\section{A Appendix}

The unital $*$-algebra $\mathcal{A}_{\theta}$ of smooth functions on the noncommutative 2 -torus consists of power series of the form

$$
a=\sum_{m, n \in \mathbb{Z}^{2}} a_{m n} U_{1}^{m} U_{2}^{n}
$$

where the (double) sequence of coefficients $\left\{a_{m n} \in \mathbb{C},(m, n) \in \mathbb{Z}^{2}\right\}$ decreases rapidly at 'infinity'

$$
\|a\|_{k}:=\sup _{m, n}\left\{\left(1+m^{2}+n^{2}\right)^{k}\left|a_{m n}\right|^{2}\right\}<\infty, \quad \forall k \in \mathbb{N} .
$$

The *-algebra structure is specified by the commutation relations of the unitary generators $U_{1}, U_{2}$

$$
U_{1} U_{2}=e^{2 \pi i \theta} U_{2} U_{1}
$$

where $\theta$ is a real parameter. 
On $A_{\theta}$ there is a (unique if $\theta$ is irrational) normalised faithful positive definite tracial state $\mathfrak{t}: \mathcal{A}_{\theta} \rightarrow \mathbb{C}$; it is given by

$$
\mathfrak{t}(a)=\mathfrak{t}\left(\sum_{(m, n) \in \mathbb{Z}^{2}} a_{m n} U_{1}^{m} U_{2}^{n}\right):=a_{00} .
$$

We call $\mathfrak{t}$ trace, for brevity. It is invariant under the action of the commutative torus $\mathbb{T}^{2}=$ $U(1) \times U(1)$ on $\mathcal{A}_{\theta}$ by automorphisms, $U_{1} \mapsto z_{1} U_{1}$ and $U_{2} \mapsto z_{2} U_{2}$, where $\left|z_{1}\right|=1=\left|z_{2}\right|$. The infinitesimal action is determined by two commuting derivations $\delta_{1}, \delta_{2}$ acting by

$$
\delta_{\mu}\left(U_{\nu}\right)=2 \pi i \delta_{\mu}^{\nu} U_{\nu}, \quad \mu, \nu=1,2 .
$$

The invariance means just that $\mathfrak{t}\left(\delta_{\mu} a\right)=0$, for $\mu=1,2$ and any $a \in \mathcal{A}_{\theta}$.

There is a sesquilinear form on $\mathcal{A}_{\theta}$

$$
(a, b)=\mathfrak{t}\left(a^{*} b\right)
$$

and a norm

$$
\|a\|:=\sqrt{\mathfrak{t}\left(a^{*} a\right)}
$$

The completion of $\mathcal{A}_{\theta}$ in this norm yields a Hilbert space $H_{\mathrm{o}}:=L^{2}\left(\mathcal{A}_{\theta}, \mathfrak{t}\right)$, which carries a $*-$ representation of $\mathcal{A}_{\theta}$ by left multiplication operators

$$
\pi(a): \quad b \mapsto a b .
$$

Moreover $\pi$ is the GNS representation w.r.t. the state $\mathfrak{t}$, the cyclic and separating vector is $1 \in \mathcal{A}_{\theta} \subset H_{\mathrm{o}}$. The operator norm of $\pi(a)$ is $\|a\|$. The associated Tomita conjugation provides an antiunitary operator $J_{\mathrm{o}}$ on $H_{\mathrm{o}}$,

$$
J_{\mathrm{o}}(b):=b^{*} .
$$

Clearly $J_{\mathrm{o}}^{2}=1$ and

$$
\pi^{\mathrm{op}}(a):=J_{\mathrm{o}} \pi\left(a^{*}\right) J_{\mathrm{o}}: \quad b \mapsto b a
$$

(right multiplication by $a$ ) is a representation of the opposite algebra of $\mathcal{A}_{\theta}$. Since the left and right multiplications commute, we have

$$
\left[\pi(a), J_{\mathrm{o}} \pi\left(b^{*}\right) J_{\mathrm{o}}\right]=0, \quad \forall a, b .
$$

(In the paper we are omitting the symbol $\pi$.)

For Dirac spinors (Hilbert space) we take the trivial module over $A_{\theta}$ of rank two

$$
H=H_{\mathrm{o}} \otimes \mathbb{C}^{2} \simeq H_{\mathrm{o}} \oplus H_{\mathrm{o}}=: H_{+} \oplus H_{-} .
$$

The inner product is $\left\langle\left(a, a^{\prime}\right),\left(b, b^{\prime}\right)\right\rangle=\mathfrak{t}\left(a^{*} b+a^{\prime} b^{*}\right)$. The charge conjugation operator on $H$ is given by

$$
J:=-i J_{\mathrm{o}} \otimes\left(\sigma_{2} \text { ○ c.c. }\right) \text {. }
$$

\section{Acknowledgements}

L.D. gratefully acknowledges the hospitality of the Institute of Physics, Jagiellonian University in Kraków. L.D. partially supported by PRIN 2010 grant "Operator Algebras, Noncommutative Geometry and Applications", A.S. partially supported by NCN grant 2012/06/M/ST1/00169. The authors express their gratitude to the referees for valuable comments. 


\section{References}

[1] Bhuyain T.A., Marcolli M., The Ricci flow on noncommutative two-tori, Lett. Math. Phys. 101 (2012), 173-194, arXiv:1107.4788.

[2] Cohen P.B., Connes A., Conformal geometry of the irrational rotation algebra, Preprint MPI (92-93), 1992.

[3] Connes A., Noncommutative geometry, Academic Press, Inc., San Diego, CA, 1994.

[4] Connes A., Moscovici H., The local index formula in noncommutative geometry, Geom. Funct. Anal. 5 (1995), 174-243.

[5] Connes A., Moscovici H., Type III and spectral triples, in Traces in Number Theory, Geometry and Quantum Fields, Aspects Math., Vol. E38, Friedr. Vieweg, Wiesbaden, 2008, 57-71, math.OA/0609703.

[6] Connes A., Moscovici H., Modular curvature for noncommutative two-tori, J. Amer. Math. Soc. 27 (2014), 639-684, arXiv:1110.3500.

[7] Connes A., Tretkoff P., The Gauss-Bonnet theorem for the noncommutative two torus, in Noncommutative Geometry, Arithmetic, and Related Topics, Johns Hopkins Univ. Press, Baltimore, MD, 2011, 141-158, arXiv:0910.0188.

[8] Dąbrowski L., Sitarz A., Curved noncommutative torus and Gauss-Bonnet, J. Math. Phys. 54 (2013), 013518, 11 pages, arXiv:1204.0420.

[9] Dạbrowski L., Sitarz A., Noncommutative circle bundles and new Dirac operators, Comm. Math. Phys. 318 (2013), 111-130, arXiv:1012.3055.

[10] Fathizadeh F., Khalkhali M., The Gauss-Bonnet theorem for noncommutative two tori with a general conformal structure, J. Noncommut. Geom. 6 (2012), 457-480, arXiv:1005.4947.

[11] Fathizadeh F., Khalkhali M., Scalar curvature for the noncommutative two torus, J. Noncommut. Geom. 7 (2013), 1145-1183, arXiv:1110.3511.

[12] Fathizadeh F., Khalkhali M., Weyl's law and Connes' trace theorem for noncommutative two tori, Lett. Math. Phys. 103 (2013), 1-18, arXiv:1111.1358.

[13] Fathizadeh F., Wong M.W., Noncommutative residues for pseudo-differential operators on the noncommutative two-torus, J. Pseudo-Differ. Oper. Appl. 2 (2011), 289-302.

[14] Lesch M., Divided differences in noncommutative geometry: rearrangement lemma, functional calculus and expansional formula, arXiv:1405.0863.

[15] Ponge R., Wang H., Noncommutative geometry and conformal geometry. III. Vafa-Witten inequality and Poincaré duality, Adv. Math. 272 (2015), 761-819, arXiv:1310.6138.

[16] Rosenberg J., Levi-Civita's theorem for noncommutative tori, SIGMA 9 (2013), 071, 9 pages, arXiv:1307.3775. 